\title{
Power-law fluctuations in phase-separated lipid membranes
}

\author{
R. Winter, ${ }^{1}$ A. Gabke, ${ }^{1}$ C. Czeslik, ${ }^{1}$ and P. Pfeifer ${ }^{2}$ \\ ${ }^{1}$ Department of Chemistry, Physical Chemistry I, University of Dortmund, Otto-Hahn-Straße 6, D-44221 Dortmund, Germany \\ ${ }^{2}$ Department of Physics, University of Missouri, Columbia, Missouri 65211
}

(Received 21 May 1999; revised manuscript received 11 August 1999)

\begin{abstract}
The spatial structure of three binary lipid mixtures, prepared as multilamellar vesicles, was studied by small-angle neutron scattering. In the fluid-gel coexistence region, large-scale concentration fluctuations appear which scatter like surface fractals for small acyl-chain mismatch and like mass fractals for large mismatch over about one decade of length. The transition is highly discontinuous: The fractal dimension of the boundary between the gel and fluid drops from 2.7 to 1.7 , the gel fraction in the fluctuations drops from about 0.5 to 0.07 , and the gel domains interlamellar correlation drops from strong to weak. We interpret the fluctuations as long-lived descendants of the incipient two-phase equilibrium state and the transition as due to changes in the gel rigidity and phase diagram. [S1063-651X(99)13912-6]

PACS number(s): 87.15.Ya, 68.55.Jk, 82.70.-y, 87.64.Bx
\end{abstract}

\section{INTRODUCTION}

Biological membranes are complex quasi-twodimensional systems composed of a large number of different lipid species and proteins [1]. Phospholipid bilayers are the basic structural component of the lipid matrix. The assembly of lipid species constituting the bilayer part can be described as a multicomponent mixture characterized by a multidimensional phase diagram with regions of phase coexistence that depend on the mixing properties of the different lipids and the thermodynamic conditions (temperature, pressure, $\mathrm{pH}$, ionic strength of solvent). It has long been conjectured that the multicomponent character of biomembranes may lead to strong membrane heterogeneity and phase segregation originating from compositional fluctuations. Fluctuations are known to be vital in biological systems under a wide variety of conditions, and they are expected to be pervasive in low-dimensional systems, such as membranes. To understand the diverse effects of the presence of several components, studies of well-defined model membranes composed of few components have to be carried out. While understanding of one-component lipid bilayers has reached a level of considerable sophistication now [2-6], this is not the case for bilayers formed from two or more lipid components.

Phase diagrams of lipid mixtures have been measured, for example, by calorimetry, x-ray diffraction, NMR, and IR spectroscopy. Typically, there is a fluid phase, a gel phase, and a fluid-gel coexistence region (phase separation), but no critical point (coalescence of the two phases). In the gel phase, the hydrocarbon chains are in a straight, elongated conformation; in the fluid phase, they are conformationally disordered (chain-melting transition). The region of interest is the two-phase region, where there is considerable controversy regarding the spatial organization and nature of the two phases [7-17]: Measurements of fluorescence recovery after photobleaching have indicated the existence of highly heterogeneous gel and fluid domains in the coexistence region [7]. Monte Carlo computer simulations of phase diagrams have exhibited long-lived percolationlike gel and fluid domains with a network of interfacial regions that have properties different from those of coexisting bulk phases [15-17].
Most recently, small-angle neutron diffraction (SANS) experiments with multilamellar vesicles have shown the presence of large-scale surface-fractal domains in the two-phase region [14].

In this paper, we report power-law fluctuations consistent with fractal behavior in the fluid-gel coexistence region, as a function of the mismatch $m$ of the acyl chains of the two lipids, defined as the ratio of the difference in chain length to the length of the shorter chain (chain lengths in units of carbon atom). We find that the fractal fluctuations occur in all three systems studied, that their size does not vary with $m$, but that their nature, including the value of the fractal dimension, undergoes an abrupt change as $m$ increases from $2 / 7$ to 0.5 . The change is from surface fractals to mass fractals, and the fractal dimension of the boundary between the gel and fluid domains drops from 2.7 to 1.7. As we will show, these results also imply a variety of other unexpected properties of the two phases: Contrary to expectations, the phase separation does not take place in each lipid bilayer independently, but produces gel and fluid domains correlated across many bilayers. Contrary to expectations, the amount and composition of the two phases differ strongly from those predicted by the equilibrium phase diagram. This provides the first direct evidence that phase separation in multilamellar membranes is dominated by long-lived nonequilibrium structures, that these structures have ramified boundaries ranging from nearly space filling to less than plane filling, and that their formation is governed by interlamellar interactions, outside the domain of single-bilayer statistical mechanics.

The mixtures investigated are DMPC/DPPC, DMPC/ DSPC, and DLPC/DSPC, with acyl chains di-C 12 (DLPC, dilauroyl-phosphatidylcholine), di- $\mathrm{C}_{14}$ (DMPC, dimyristoylphosphatidylcholine), di-C 16 (DPPC, dipalmitoylphosphatidylcholine), and $\mathrm{di}_{18} \mathrm{C}_{18}$ (DSPC, distearoylphosphatidylcholine). Thus both components are saturated phospholipids, the mismatches are $m=1 / 7,2 / 7,1 / 2$, the middle system is the one where surface-fractal fluctuations were first observed and studied as a function of pressure [14], and the last two systems are the ones where phase separation in a single bilayer was simulated [15-17]. Figure 

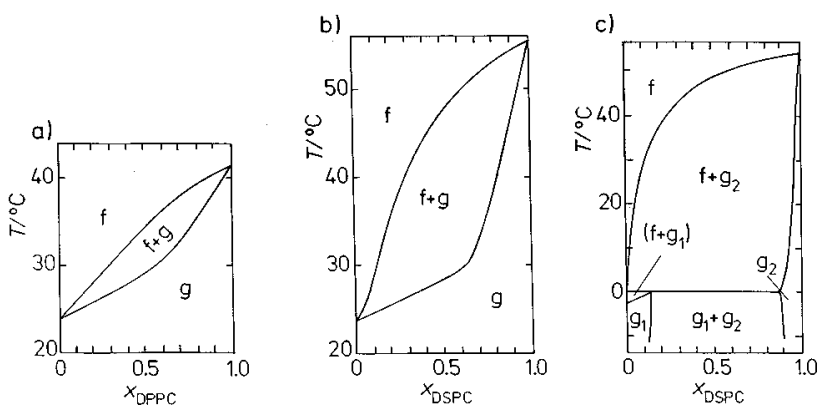

FIG. 1. T,x-phase diagram of the lipid mixtures (a) DMPC/ DPPC (di-C $14 /$ di-C $\left.\mathrm{C}_{16}\right)$, (b) DMPC/DSPC $\left(\right.$ di-C $_{14} / \mathrm{di}_{-} \mathrm{C}_{18}$ ), and (c) DLPC/DSPC ( di- $\left.\mathrm{C}_{12} / \mathrm{di}^{-\mathrm{C}_{18}}\right)$, in excess water, as determined from heat-capacity, x-ray diffraction, and NMR experiments $(g$, gel; $f$, fluid; $x$, mole fraction).

1 shows the phase diagrams of the three mixtures [2,6,9]. The narrow fluid-gel coexistence region in DMPC/DPPC signals nearly ideal mixing of the two components (isomorphous system). The coexistence region in DMPC/DSPC by comparison is broad and manifests pronounced departures from ideality. In both systems, the components are completely miscible in the all-gel and all-fluid phase. The strongest departure from ideality occurs in DLPC/DSPC, where the acyl-chain mismatch is so large that the components are essentially immiscible in the gel phase ( gel $_{1}$-gel ${ }_{2}$ phase, peritectic behavior) at low temperature. As the temperature is raised and the three-phase line is crossed, the gel ${ }_{1}$ phase, consisting mainly of DLPC, melts, and a fluid-gel ${ }_{2}$ phase forms.

To observe the fluctuations, we used SANS with H/D contrast variation $[14,18,19]$. The large difference in the coherent scattering length of hydrogen $\left(b_{\mathrm{H}}=-0.374\right.$ $\left.\times 10^{-12} \mathrm{~cm}\right)$ and deuterium $\left(b_{\mathrm{D}}=0.667 \times 10^{-12} \mathrm{~cm}\right)$ provides an excellent contrast when one of the components is deuterated.

\section{MATERIALS AND METHODS}

Equimolar mixtures of $\operatorname{DMPC}\left(d_{54}\right) / \mathrm{DPPC}$, $\operatorname{DMPC}\left(d_{54}\right) / \mathrm{DSPC}$, and $\operatorname{DLPC} / \operatorname{DSPC}\left(d_{70}\right)$ were prepared by dissolving the protonated and deuterated lipids (Avanti Polar Lipids, Birmingham, AL) in chloroform. The solvent was removed using a rotary evaporator, and the samples were lyophilized for several hours. Homogeneous samples consisting of multilamellar vesicles were obtained after several freeze-thaw-vortex cycles. The composition of the $\mathrm{H}_{2} \mathrm{O} / \mathrm{D}_{2} \mathrm{O}$ mixture for the dispersion of the vesicles, with a lipid mass fraction of about $30 \%$, was adjusted so that the scattering cross section density of $\mathrm{H}_{2} \mathrm{O} / \mathrm{D}_{2} \mathrm{O}$ was equal to that of a homogeneous mixture of the lipid components. Under these so-called matching conditions, scattering arises essentially from the inhomogeneous distribution of the lipid components in the vesicle.

The diffraction patterns were recorded starting from the all-fluid phase of the lipid systems. Typically, for each temperature the measurements (at two detector positions) took 3 h. After a new temperature was adjusted, the sample was allowed to equilibrate for at least $15 \mathrm{~min}$. To ensure that a new lamellar phase has indeed been formed within the time scale of the experiment and that the transitions are reversible, we recorded the lamellar $d$ spacings by small-angle $\mathrm{x}$-ray diffraction. In a time-resolved x-ray diffraction measurement on the all-fluid-gel-fluid two-phase transition of the system DMPC/DSPC (1:1) using synchrotron radiation in combination with the pressure-jump relaxation technique, we found that the transition is indeed complete within about $30 \mathrm{~s}$.

The scattering experiments were performed on the D11 diffractometer at the Institut Laue-Langevin, Grenoble, and the KWS2 diffractometer at the Forschungszentrum Jülich. The range of momentum transfers $Q=(4 \pi / \lambda) \sin \Theta$ (scattering angle $2 \Theta$, wavelength of radiation $\lambda$ ) was $3.3 \times 10^{-3}-1$ $\times 10^{-1} \AA^{-1}$, covering a range of lengths of $60-1900 \AA$ $(2 \pi / Q)$. A standard treatment of isotropic SANS data has been performed [20]. Briefly, the measured intensity distributions have been corrected for absorption, sample thickness, inelasticity, and background.

The differential scattering cross section per unit volume of the sample can be written as

$$
\frac{d \Sigma}{d \Omega}=n_{p}(\Delta \rho)^{2} V_{p}^{2} P(Q) S(Q),
$$

where $n_{p}$ denotes the number density of particles (lipid molecules), $V_{p}$ the particle volume, and $\Delta \rho=\rho_{p}-\rho_{s}$ the contrast, i.e., the difference in mean scattering length density of the particles $\left(\rho_{p}\right)$ and the solvent $\left(\rho_{s}\right) . \quad P(Q)$ is the form factor of the particles, and $S(Q)$ is the structure factor describing the spatial distribution of the particles. By setting $P(Q)=1$, we treat the molecules as point particles and the sample as consisting of two phases, each of which has a constant scattering length density, described by $S(Q)$. This treatment is appropriate because we analyze data only at $Q$ $<0.03 \AA^{-1}$.

For the structure factor, we consider the following three expressions:

$$
\begin{gathered}
S(Q)=1+\frac{A n_{p} k_{B} T \chi_{T}+B^{2} n_{p}^{2} S_{\mathrm{cc}}(0)-1}{1+(Q \xi)^{2}}, \\
S(Q)=N \min \left\{1,(Q \xi)^{-D_{m}}\right\}, \\
S(Q)=N \min \left\{1,(Q \xi)^{D_{s}-6}\right\} .
\end{gathered}
$$

Equation (2) is the Ornstein-Zernike equation for scattering from equilibrium fluctuations near a critical point [2123 ], with $S_{\mathrm{cc}}(0)$ the concentration-concentration structure factor, $\chi_{T}$ the isothermal compressibility, $\xi$ the correlation length, $A$ and $B$ constants involving the scattering lengths and partial molar volumes of the components, and range of validity $Q \xi<6$ [for $\xi^{-1}<Q<V_{p}^{-1 / 3}$, one has $S(Q)$ $\propto Q^{-2+\eta}$ with $\left.\eta \approx 0.03\right]$ [22]. The motivation for considering Eq. (2) is that the chain-melting transition in singlecomponent phospholipids, although of first order, is probably close to a critical point $[24,25]$, which suggests that the transition in mixtures may be close to a critical point, too, and exhibits Ornstein-Zernike fluctuations.

Equations (3) and (4) describe scattering from fluctuations that are mass fractals and surface fractals, with mass-fractal dimension $D_{m}$ and surface-fractal dimension $D_{s}$, respectively [26-31]. $\quad N$ is the number of particles per domain, $\xi$ 
is the domain diameter, and the range of validity is $Q a \ll 1$, with $a$ the lower cutoff of the fractal regime. The crossover from power-law scattering for $\xi^{-1} \ll Q \ll a^{-1}$ to constant scattering for $Q \ll \xi^{-1}$ is treated as sharp in Eqs. (3) and (4), and the length $\xi$ in the two power laws is accurate within a factor of order 1. Together, Eqs. (3) and (4) represent a family of fluctuation geometries, parametrized by the pair $\left(D_{m}, D_{s}\right)$, subject to

$$
\begin{gathered}
0<D_{m} \leqslant 3, \\
0 \leqslant D_{m}-D_{s} \leqslant 1, \\
\left(3-D_{m}\right)\left(D_{m}-D_{s}\right)=0,
\end{gathered}
$$

in which the space-filling ability of the domains increases as $D_{m}$ and $D_{m}-D_{s}$ increase: For $0<D_{m}<3$, the domains are tenuous clusters whose surface scales as the mass (mass fractal, $\left.D_{s}=D_{m}\right)$. For $D_{m}=3$, the domains are compact clusters whose surface scales independently of the mass (surface fractal, $2 \leqslant D_{s} \leqslant 3$ ), are marginally space filling when $D_{s}$ $=3$ (maximally convoluted surface), and are maximally space filling when $D_{s}=2$ (smooth surface). Taking $D_{m}$ and $D_{s}$ as independent parameters subject to Eqs. (5) and clusters as compact at lengths below $a$, one can combine Eqs. (3) and (4) into the single expression

$$
S(Q)=\left(\frac{\xi}{a}\right)^{D_{m}} \frac{a^{3}}{V_{p}} \min \left\{1,(Q \xi)^{-D_{m}}(Q \xi)^{-\left(D_{m}-D_{s}\right)}\right\}
$$

(see also [30]), which decomposes $S(Q)$ into a factor describing the bulk structure (mass distribution) of the domain, $(Q \xi)^{-D_{m}}$, and a factor describing the surface structure relative to the bulk, $(Q \xi)^{-\left(D_{m}-D_{s}\right)}$. The decomposition is analogous to that of $d \Sigma / d \Omega$ into the structure and form factor, reflecting the presence of a structural hierarchy. In Eq. (1), the hierarchy is that particles form subunits of domains; in Eq. (6), it is that the surface forms a substructure of the bulk. Since $D_{m}>D_{m}-D_{s}$, the bulk term always dominates over the surface term. Equation (6) provides a unified picture that the scattered intensity falls off with $Q$ increasingly fast as the space-filling ability of the domains increases.

\section{EXPERIMENTAL RESULTS}

$D M P C / D P P C$. From differential scanning calorimetry it is known that deuteration of DMPC lowers the transition of DMPC by $4{ }^{\circ} \mathrm{C}$ and shifts the two-phase region of $\operatorname{DMPC}\left(d_{54}\right) / \mathrm{DPPC}$ to $26-34^{\circ} \mathrm{C}$. Figure 2 presents SANS data of the mixture at different temperatures: below (12, $\left.20^{\circ} \mathrm{C}\right)$, in $\left(31^{\circ} \mathrm{C}\right)$, and above $\left(48^{\circ} \mathrm{C}\right)$ the two-phase region. Almost zero scattering intensity is observed at low and high $T$, in agreement with a homogeneous mixture of the two components leading to $\Delta \rho=0$ in Eq. (1). The intensity increases significantly in the two-phase region. No Porod slope of -4 is observed at large $Q$ [Eq. (4) with $D_{s}=2$ ], where the scattering is governed by the small-scale structure of the fluctuations. Thus the fluctuations do not have smooth surfaces. Neither do they follow the Ornstein-Zernike law (2) [plots of $(d \Sigma / d \Omega)^{-1}$ vs $Q^{2}$ ], consistent with the absence of a critical point in the phase diagram (horizontal tangent of liquidus or solidus line). Instead, they give rise to power-law
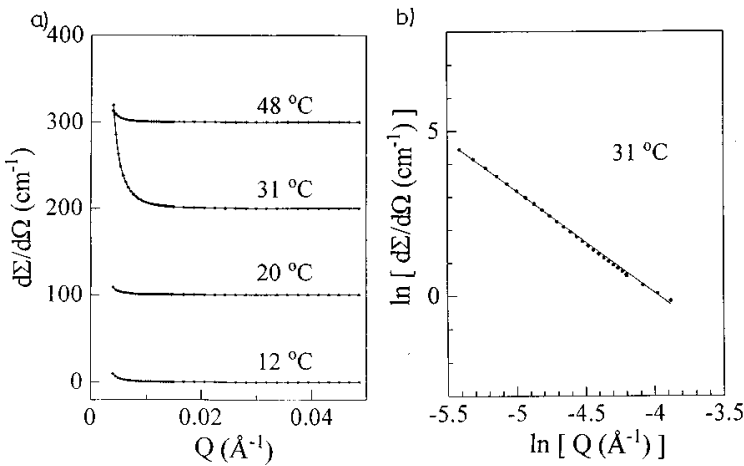

FIG. 2. (a) SANS curves of DMPC $\left(d_{54}\right) / \mathrm{DPPC}$ at $x_{\mathrm{DPPC}}=0.5$ (aqueous dispersion). The curves at different temperatures are shifted by $100 \mathrm{~cm}^{-1}$ relative to each other. (b) Double-logarithmic plot of the curve for $31^{\circ} \mathrm{C}$.

scattering over the whole $Q$ range covered [Fig. 2(b)], i.e., over distances of 300-1400 $\mathrm{A}$. The exponent from the log$\log$ plot is $-3.1 \pm 0.1$, consistent with surface-fractal fluctuations of dimension $D_{s}=2.9 \pm 0.1$ [Eq. (4)]. The absence of a crossover at both ends of the power-law regime gives $a<300 \AA$ and $\xi>1400 \AA$.

$D M P C / D S P C$. As above, deuteration of DMPC shifts the solidus line of the two-phase region of $\operatorname{DMPC}\left(d_{54}\right) / \mathrm{DSPC}$ to slightly lower temperatures. Figure 3 shows the scattering curves of the mixture for $T=21-80^{\circ} \mathrm{C}$. Again, the scattering intensity is significant only in the two-phase region, i.e., for $T=26-45^{\circ} \mathrm{C}$. Again, the data do not obey the OrnsteinZernike law in the fluid-gel coexistence region, but a power law over the whole $Q$ range covered (Fig. 4), corresponding to distances of $240-1200 \AA$. The exponent is $-3.3 \pm 0.1$ for all five temperatures, consistent again with surface-fractal fluctuations, here with $D_{s}=2.7 \pm 0.1$.

$D L P C / D S P C$. The three-phase line of DLPC/DSPC $\left(d_{70}\right)$ is at $2{ }^{\circ} \mathrm{C}$ and the fluid-gel coexistence region is at $2-51{ }^{\circ} \mathrm{C}$ $\left(0-48^{\circ} \mathrm{C}\right.$ for protonated DLPC/DSPC, Fig. 1). Figure 5(a) shows the scattering curves at temperatures $-5-60^{\circ} \mathrm{C}$. As in the other mixtures, the scattering intensity is almost zero at temperatures above the liquidus line and increases as the temperature drops into the extended fluid-gel ${ }_{2}$ coexistence region. Unlike in the other mixtures, however, significant scattering is also observed in the gel $_{1}$ - gel $_{2}$ coexistence region (below $2{ }^{\circ} \mathrm{C}$ ). The $\log$-log plots [Fig. 5(b)] exhibit straight lines at temperatures well inside the fluid-gel ${ }_{2}$ region, but not

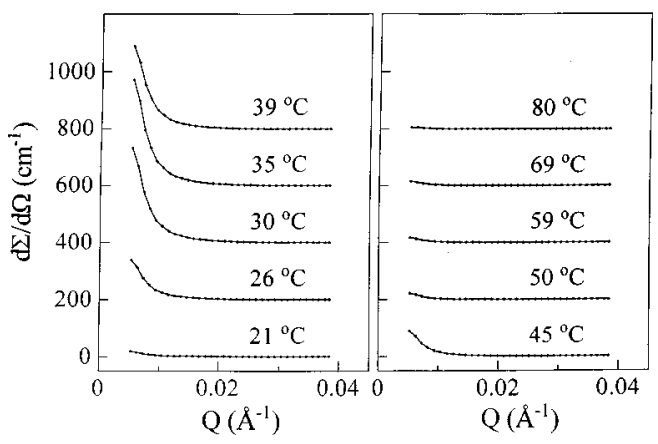

FIG. 3. SANS curves of $\operatorname{DMPC}\left(d_{54}\right) / \mathrm{DSPC}$ at $x_{\mathrm{DSPC}}=0.5$ (aqueous dispersion). The curves at different temperatures are shifted by $200 \mathrm{~cm}^{-1}$ relative to each other. 


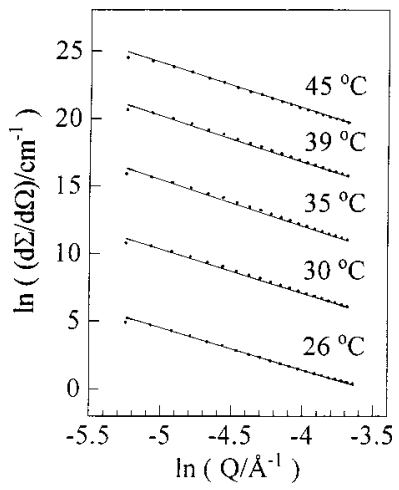

FIG. 4. In-ln plots of selected curves in Fig. 3, shifted by $\ln \left[d \Sigma / d \Omega\left(\mathrm{cm}^{-1}\right)\right]=5$.

in the gel $_{1}-$ gel $_{2}$ region and close to the three-phase line in the fluid-gel ${ }_{2}$ region. The plots well inside the fluid-gel ${ }_{2}$ region yield a slope of $-1.7 \pm 0.2$, consistent with mass-fractal fluctuations of dimension $D_{m}=1.7 \pm 0.2$ [Eq. (3)]. The range of length scales is $230-1800 \AA$ at $25^{\circ} \mathrm{C}$. At -5 and $5^{\circ} \mathrm{C}$, close to the three-phase line, the linear regimes are too short to support a fractal structure.

\section{DISCUSSION AND CONCLUSIONS}

The structure of the fluctuations inferred from the scattering data of the three systems is summarized in Table I. The most prominent features are the following.

(i) A typical fluid or gel domain is not confined to an individual bilayer, but spans many bilayers: The bilayers in which phase separation occurs are stacked in concentric lamellae of about $70 \AA$ thickness (including a water layer of $10-20 \AA$ between every pair of bilayers) in a vesicle of about $1 \mu \mathrm{m}$ diameter. Were the domains islands in individual bilayers uncorrelated with islands in neighboring bilayers, the vesicle would be homogeneous at scales above, say, $200 \AA$ (three bilayers) regardless of the lateral size of the islands, and the scattered intensity at $Q<2 \pi /(200 \AA)$ would vanish. Thus the fractals are interlamellar, not intralamellar, and involve strong interactions between bilayers. The number of bilayers participating in a domain is $\xi /(70 \AA)=17-26$. In a
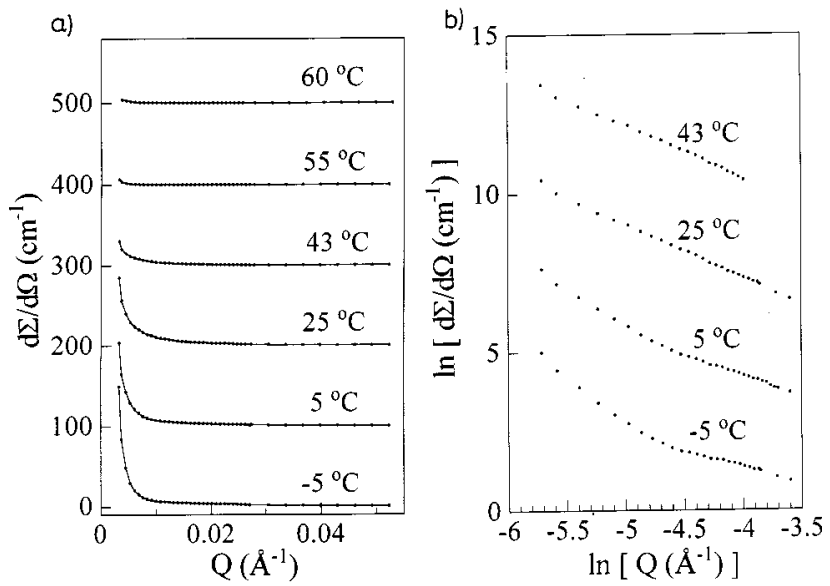

FIG. 5. SANS curves of DLPC/DSPC $\left(d_{70}\right)$ at $x_{\mathrm{DSPC}}=0.5$ (aqueous dispersion). The curves in (a) are shifted by $100 \mathrm{~cm}^{-1}$ and those in (b) by $\ln \left[d \Sigma / d \Omega\left(\mathrm{cm}^{-1}\right)\right]=5$ relative to each other.
TABLE I. Fractal properties of the fluctuations in the fluid-gel coexistence region of binary phospholipid mixtures (error bars in $a$, $\xi \sim 10 \%)$.

\begin{tabular}{lccc}
\hline \hline & DMPC/DPPC & DMPC/DSPC & DLPC/DSPC \\
\hline$m$ & $1 / 7$ & $2 / 7$ & $1 / 2$ \\
$D_{m}$ & 3 & 3 & $1.7 \pm 0.2$ \\
$D_{s}$ & $2.9 \pm 0.1$ & $2.7 \pm 0.1$ & $1.7 \pm 0.2$ \\
$a(\AA)$ & $<300$ & $<240$ & $<230$ \\
$\xi(\AA)$ & $>1400$ & $>1200$ & $\sim 1800$ \\
\hline \hline
\end{tabular}

single bilayer, the phase-separated region is a $\left(D_{m}-1\right)$-dimensional island with a $\left(D_{s}-1\right)$-dimensional boundary.

(ii) The scaling regime is as extended as it can be for fluctuations between one bilayer $(a>70 \AA)$ and one vesicle radius $(\xi<5000 \AA$; the aqueous vesicle center excludes larger fluctuations). We believe this makes the domains reasonably well-defined fractals. The surface-fractal structure of the domains changes only sightly as the chain mismatch $m$ increases from $1 / 7$ to $2 / 7$ and then switches to a mass-fractal structure at $m=1 / 2$. The discontinuity is particularly striking when the results are plotted on the curve of all possible pairs $\left(D_{m}, D_{s}\right)$ : The space-filling ability of the domains first increases slightly and then drops drastically (Fig. 6). The value $D_{m}=1.7 \pm 0.2$ at $m=1 / 2$ is remarkably close to the massfractal dimension observed in the phase separation of a solution of micelles $\left(\mathrm{C}_{12} \mathrm{E}_{6}\right.$ in $\left.\mathrm{D}_{2} \mathrm{O}\right), D_{m}=1.6 \pm 0.1$, quenched at its critical point [19]. The coincidence is remarkable because the growth mechanism of the two fractals is presumably very different.

(iii) The fractal structure completely specifies the pair correlation function of a single domain,

$$
g(r)=\max \left\{\left(\frac{a}{r}\right)^{3-D_{m}}-\left(\frac{a}{\xi}\right)^{3-D_{m}}\left(\frac{r}{\xi}\right)^{3-D_{s}}, 0\right\},
$$

defined as the angular average of $\int \rho(\vec{x}) \rho(\vec{x}$ $+\vec{r}) d^{3} x / \int \rho(\vec{x}) d^{3} x$, where $\rho(\vec{x})=1$ if $\vec{x}$ is in the domain and

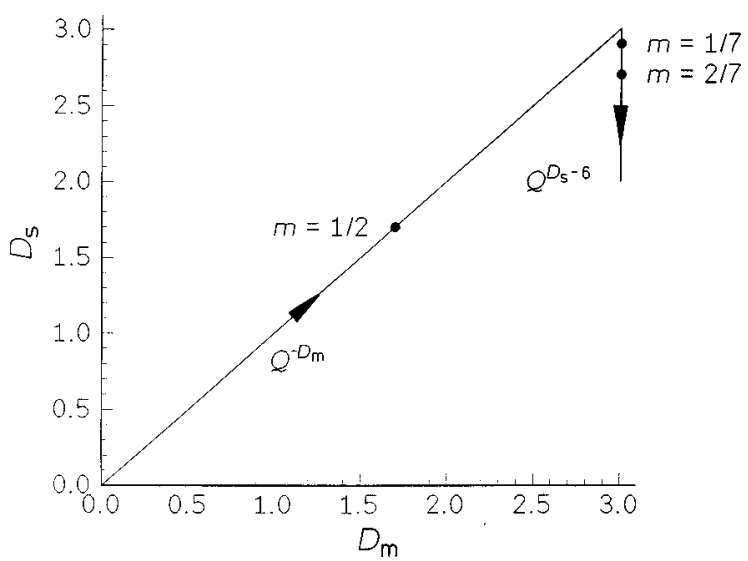

FIG. 6. Experimental values of $\left(D_{m}, D_{s}\right)$ (dots) on the curve of all solutions $\left(D_{m}, D_{s}\right)$ of Eqs. (5) (solid line). The $45^{\circ}$ line is the locus of mass fractals; the vertical line is the locus of surface fractals. Arrows indicate increasing space-filling ability of the fluctuations. 
$\rho(\vec{x})=0$ otherwise. Equation (7), valid for $r \gg a$, combines the power law $g(r)=(r / a)^{D_{m}-3}$ for mass fractals $(r \gg a)$ $[27,28], g(r)=1-(r / \xi)^{3-D_{s}}$ for surface fractals $(r \ll \xi)$ [29-31], and the condition $g(r)=0$ for $r \geqslant \xi$. It quantifies the correlation of islands in different bilayers belonging to the same domain. The correlation is strong or weak if $g(r)$ falls off slowly or rapidly, respectively. The correlation drops from strong $[g(r) \sim 1$ for $m=1 / 7,2 / 7]$ to weak $[g(r)$ $\sim(r / a)^{-1.3}$ for $\left.m=1 / 2\right]$ in the transition from surface to mass fractals.

The picture that emerges for the three systems is as follows. For small chain mismatch, $m=1 / 7,2 / 7$, the two lipids are sufficiently alike to interact attractively (miscibility in the gel phase, small hydrophobic mismatch energy term in the Hamiltonian). The fluid and gel compositions in the twophase region do not differ widely, and one expects comparable volume fractions of gel and fluid, $\phi$ and $1-\phi$, in a fluctuation volume of $\xi^{3}$ (e.g., $\phi=0.6$ for DMPC/DPPC at $31{ }^{\circ} \mathrm{C}$, Fig. 1). This is what we find: Since the complement of a surface fractal is also a surface fractal, both phases form domains with $D_{m}=3$ and nominally $\phi=1-\phi$. In fact, Eq. (4) is equivalent to scattering from a distribution of independent droplets of one phase in the other, with the number of droplets of radius $\geqslant r$ equal to $(r / \xi)^{-D_{s}}$ (pore-size distribution of a fractal surface) [29-31]. The droplet picture says that the fluid and gel alternate so that within distance $2 r$ from a fluid segment of diameter $2 r$ there is typically a gel segment of diameter $2 r$. The high surface dimension $D_{s}$ $=2.7-2.9$ (strongly meandering interface) implies a low fluid-gel surface tension.

This symmetry between the fluid and gel phases is broken at $m=1 / 2$. The large mismatch causes the two lipids to interact repulsively (immiscibility of gel $_{1}$ and $\mathrm{gel}_{2}$, large hydrophobic mismatch energy term in the Hamiltonian [17]) and separate into one phase with $D_{m}=1.7$ and the other with $D_{m}=3$ in the fluid-gel ${ }_{2}$ coexistence region. Let us assume the minority phase $D_{m}=1.7$ is the gel phase and pure DSPC. For the gel phase we then have $\phi=(a / \xi)^{3-D_{m}} \approx 0.07$ and $x_{\mathrm{DSPC}, \mathrm{gel}}=1.0$, and for the fluid phase $x_{\mathrm{DSPC} \text {,fluid }}=(1$ $-2 \phi) /(2-2 \phi) \approx 0.46$. In comparison, the phase diagram gives $\phi=0.48, x_{\mathrm{DSPC}, \text { gel }}=0.95, x_{\mathrm{DSPC} \text {,fluid }}=0.07$ at $25^{\circ} \mathrm{C}$. Thus, the gel fraction $\phi$ and mole fraction $x_{\mathrm{DSPC} \text {,fluid }}$ from SANS are far from the equilibrium values at $25^{\circ} \mathrm{C}$. Instead, they are close to the equilibrium values at $48^{\circ} \mathrm{C}$, where the two-phase region meets the all-fluid region (incipient twophase equilibrium state). We interpret the domains at $25^{\circ} \mathrm{C}$ as nonequilibrium descendants of the incipient two-phase equilibrium state: They start as tiny gel clusters at $48^{\circ} \mathrm{C}(\phi$ $\approx 0, x_{\mathrm{DSPC}, \mathrm{gel}}=0.99$ ) and grow as we lower the temperature (all samples were prepared from the all-fluid phase). For a DSPC cluster to grow, the chain of a neighboring DSPC molecule in the fluid must switch from contracted to elongated. But DSPC is the longer of the two lipids, and to make it even longer in the presence of short, fluid DLPC's requires voids which are produced only infrequently by thermal motion. The rigidity of the gel makes such voids especially rare adjacent to a cluster wall and hence inside the convex hull of the cluster. This favors growth at the cluster tip and leads to mass fractals. It may, in fact, also lead to surface fractals, if the gel is less rigid (allowing voids to form at all surface sites equally) or if the gel incorporates also the shorter of the two lipids. Both conditions are met by the other two mixtures, so the domains for $m=1 / 7,2 / 7$ may be viewed as offspring of incipient two-phase states, too.

The model accounts for the nonequilibrium structure of the fluctuations in terms of the interaction between short and long lipids and the corresponding phase diagrams. It has no time dependence because the SANS data showed no time dependence (over periods of up to $15 \mathrm{~h}$ ). It is an irreversible growth model for a metastable state, addressing a scenario very different from the time-dependent droplet growth in early-late stage growth theories (approach to equilibrium) [32]. Additional experiments will have to be carried out to see over what time scales, if any, the fluctuations decay. Additional work will have to be done to test our growth model, e.g., in relation to recent simulations of stacks of one-component bilayers [33]. In terms of biological consequences, the fluctuations are expected to control the diffusion of membrane components, water permeability, membrane potential, rate of fusion of adjacent bilayers, and conformation of embedded proteins, to name a few.

\section{ACKNOWLEDGMENTS}

We thank G. Meier for assistance with the SANS experiments in Jülich and P. W. Schmidt for helpful discussions. We gratefully acknowledge financial support from the Deutsche Forschungsgemeinschaft and the Petroleum Research Fund, administered by the American Chemical Society.
[1] P. Yeagle, The Structure of Biological Membranes (CRC, Boca Raton, FL, 1992).

[2] G. Cevc and D. Marsh, Phospholipid Bilayers (Wiley, New York, 1987).

[3] Phospholipids Handbook, edited by G. Cevc (Dekker, New York, 1993).

[4] Structure and Dynamics of Membranes, edited by R. Lipowsky and E. Sackmann (Elsevier, Amsterdam, 1995), Vols. 1A and $1 \mathrm{~B}$.

[5] R. Winter and W.-C. Pilgrim, Ber. Bunsenges. Phys. Chem. 93, 708 (1989).

[6] R. Winter, J. Erbes, C. Czeslik, and A. Gabke, J. Phys.: Condens. Matter 10, 11499 (1998).
[7] T. Bultmann, W. L. C. Vaz, E. C. C. Melo, R. B. Sisk, and T. E. Thompson, Biochemistry 30, 5573 (1991).

[8] P. F. F. Almeida, W. L. C. Vaz, and T. E. Thompson, Biochemistry 31, 7198 (1992).

[9] S. Mabrey and J. M. Sturtevant, Proc. Natl. Acad. Sci. USA 73, 3862 (1976).

[10] G. Schmidt and W. Knoll, Ber. Bunsenges. Phys. Chem. 89, 36 (1985).

[11] M. R. Morrow, R. Srinivasan, and N. Grandal, Chem. Phys. Lipids 58, 63 (1991).

[12] M. B. Sankaram and T. E. Thompson, Biochemistry 31, 8258 (1992).

[13] A. Landwehr and R. Winter, Ber. Bunsenges. Phys. Chem. 98, 
1585 (1994).

[14] C. Czeslik, J. Erbes, and R. Winter, Europhys. Lett. 37, 577 (1997).

[15] M. Bloom, E. Evans, and O. Mouritsen, Q. Rev. Biophys. 24, 293 (1991).

[16] K. Jørgensen, M. M. Sperotto, O. G. Mouritsen, J. H. Ipsen, and M. J. Zuckermann, Biochim. Biophys. Acta 1152, 135 (1993).

[17] K. Jørgensen and O. G. Mouritsen, Biophys. J. 95, 942 (1995).

[18] W. Knoll, J. Haas, H. B. Stuhrmann, H.-H. Füldner, H. Vogel, and E. Sackmann, J. Appl. Crystallogr. 14, 191 (1981).

[19] J. P. Wilcoxon, D. W. Schaefer, and E. W. Kaler, Phys. Rev. Lett. 60, 333 (1988).

[20] Neutron X-ray and Light Scattering: Introduction to an Investigative Tool for Colloidal and Polymeric Systems, edited by P. Lindner and Th. Zemb (North-Holland, Amsterdam, 1991).

[21] H. E. Stanley, Introduction to Phase Transitions and Critical Phenomena (Clarendon, Oxford, 1971).

[22] M. Chandrasekhar and P. W. Schmidt, Phys. Rev. B 25, 6730 (1982).

[23] P. Damay, F. Leclercq, and P. Chieux, J. Phys. Chem. 88,
3734 (1984).

[24] R. Zhang, W. Sun, S. Tristram-Nagle, R. L. Headrick, R. M. Suter, and J. F. Nagle, Phys. Rev. Lett. 74, 2832 (1995).

[25] J. Lemmich, K. Mortensen, J. H. Ipsen, T. Hønger, R. Bauer, and O. G. Mouritsen, Phys. Rev. Lett. 75, 3958 (1995).

[26] P. Pfeifer and M. Obert, in The Fractal Approach to Heterogeneous Chemistry-Surface, Colloids, Polymers, edited by D. Avnir (Wiley, Chichester, 1989), pp. 11-43.

[27] T. Freltoft, J. K. Kjems, and S. K. Sinha, Phys. Rev. B 33, 269 (1986).

[28] J. Teixeira, J. Appl. Crystallogr. 21, 781 (1988).

[29] H. D. Bale and P. W. Schmidt, Phys. Rev. Lett. 53, 596 (1984).

[30] P. Pfeifer, in Multiple Scattering of Waves in Random Media and Random Rough Surfaces, edited by V. V. Varadan and V. K. Varadan (Pennsylvania State University, University Park, 1987), pp. 45-48.

[31] P. Pfeifer, Springer Ser. Surf. Sci. 10, 283 (1988).

[32] E.g., J. D. Gunton, M. San Miguel, and P. S. Sahni, in Phase Transitions and Critical Phenomena, edited by C. Domb and J. L. Lebowitz (Academic, London, 1983), Vol. 8, pp. 267-482.

[33] N. Goullaev and J. F. Nagle, Phys. Rev. Lett. 81, 2610 (1998). 